\title{
Extending the global workspace theory to emotion: Phenomenality without access
}

\author{
Dennis J.L.G. Schutter* and Jack van Honk \\ Affective Neuroscience Section, Helmholtz Research Institute, Utrecht University, Utrecht, The Netherlands \\ Received 17 February 2004 \\ Available online 17 June 2004
}

\begin{abstract}
Recent accounts on the global workspace theory suggest that consciousness involves transient formations of functional connections in thalamo-cortico-cortical networks. The level of connectivity in these networks is argued to determine the state of consciousness. Emotions are suggested to play a role in shaping consciousness, but their involvement in the global workspace theory remains elusive. In the present study, the role of emotion in the neural workspace theory of consciousness was scrutinized by investigating, whether unconscious (masked) and conscious (unmasked) display of emotional compared to neutral facial expressions would differentially modulate EEG coherence. EEG coherence was measured by means of computing an average EEG coherence value between the frontal, parietal, and midline scalp sites. Objective awareness checks evidenced that conscious identification of the masked facial expressions was precluded. Analyses revealed reductions in EEG coherence in the lower frequency range for the masked as compared to unmasked neutral facial expressions. Crucially, a decline in EEG coherence was not observed for the emotional facial expressions. In other words, the level of EEG coherence did apparently vary as a function of awareness, but not when emotion was involved. The current finding suggests that EEG coherence is modulated by unconscious emotional processes, which extends common views on the global workspace architecture of consciousness.
\end{abstract}

(c) 2004 Elsevier Inc. All rights reserved.

Keywords: EEG; Coherence; Global workspace theory; Emotion; Consciousness

\footnotetext{
${ }^{*}$ Corresponding author. Fax: +31-30-253-4511.

E-mail address: d.schutter@fss.uu.nl (D.J.L.G. Schutter).
} 


\section{Introduction}

The advent of the modern neurosciences resulted in a regained interest in studying the bodymind problem, this time particularly directed at the unravelment of the neural correlates of consciousness (NCC) (Crick \& Koch, 1998). A popular and valuable heuristic model for studying the NCC is the global workspace theory (GWT). The GWT is a cognitive architecture, which suggests that consciousness involves the integration of separate unconscious specialized networks (modules) widely distributed throughout the brain (Baars, 1988, 2002). Some of these unconscious networks, designated as contexts, are suggested to be capable of shaping conscious contents (Baars \& Franklin, 2003). Among these contexts in the GWT, the subcortical emotional system is argued to play an important role in constraining consciousness. Whereas some theorists have suggested that emotions are occurring after disturbances whenever cognitive processing is hampered (e.g., Oately, 1992), others have advocated the view that emotions originate from biological systems shaped throughout evolution (Buck, 1999; Tomkins, 1982). Moreover, the emotional system is capable of amplifying other mechanisms of behavior, such as cognition (Tomkins, 1982), fueling the organism with drives and intentions. In particular, this amplification principle can be associated with several qualitative aspects of consciousness, the so-called qualia.

Albeit the GWT of consciousness is a functional architecture, it can provide for a neurophysiologically inspired framework. In this light, the global workspace might be viewed as a cortical landscape where consciousness evolves from ordered synchronized activity in different cortical modules into a transient metastable cortical state of global activity. The term metastability was introduced by Kelso (1984) and further developed by Fingelkurts and Fingelkurts (2001), referring to a situation where there are neither stable nor unstable states, only coexisting tendencies. According to Dehaene, Kerszberg, and Changeux (1998), information becomes conscious if the involved neural network is amplified by top-down amplification into a global brainscale state of coherent activity. Furthermore, Dehaene and Naccache (2001) synthesize that the neural architecture of the GWT can be captured as follows:

“... a distributed neural system or 'workspace' with long-distance connectivity that can potentially interconnect multiple specialized brain areas in a coordinated, though variable manner." (p. 13)

In other words, the physical realization of consciousness might be interpreted in terms of distributed, but integrated processing, which Tononi and Edelman (1998) call global mapping. Moreover, according to the neural workspace framework of Dehaene, Sergent, and Changeux (2003), consciousness can only arise when the stimulus duration is long enough to cause alterations in neural activity that initiate the formation of a metastable brain state. Tononi and Edelman (1998) have argued that the intricacy and level of activation in thalamo-cortico-cortical (TCC) networks are essential in the generation of consciousness. In particular, the TCC networks model seem to provide for a perfectly reasonable neurophysiological architecture for Baars (1988) GWT wherein a 'central information exchange' is continuously receiving and binding its afferent projections from input processors into coherent units (Zeman, 2001). Furthermore, information streams do not only flow in thalamo-cortical (vertical) direction, but also in cortico-cortical (horizontal) direction. On the level of physical realization, the transient formation of functional spatio-temporal synchronization is argued to be responsible for constructing these TCC network units (Crick \& Koch, 1998). The cortical regions reflect the input processors, while the thalamus acts as the central information exchanger or gating structure (Llinas, Ribrary, Contreras, 
\& Pedroarena, 1998). Since the cortical mantle is organized into discrete macrocolumns, it might provide for a physical basis of the input processors units in the GWT (Dehaene et al., 2003). This notion is in line with the ideas of Fingelkurts and Fingelkurts (2001), who have argued that metastable operational modules may give rise to a stream of consciousness. Thus, from the current perspective, the cortex can be viewed as an epigenetic landscape in which transient shortlived functional connectivity between cortical macrocolumns give rise to consciousness (Fig. 1).

The fact that this does need to be an all-or-none phenomenon per se is conceptualized by Block (1995, 1996) who distinguishes two concepts of consciousness: phenomenal and reflexive consciousness. Phenomenal consciousness represents the mere experience of a stimulus, whereas reflexive consciousness is argued to be readily accessible to self-report, reasoning and action. From this viewpoint "unconscious" percepts can, according to Block (2001), be explained in two possible ways: (1) the unconscious percepts are both phenomenally and reflexively innate or (2) the unconscious percepts are phenomenally conscious, but remain reflexively unconscious. Thus, although information can be permanently inaccessible phenomenally and reflexively, "unseen" stimuli can render a more temporal disclosure to consciousness. If a stimulus is presented for a very short duration (e.g., $14 \mathrm{~ms}$ ) and followed by a mask, participants are not able to consciously access the information to which they were exposed to, but careful behavioral assessment can reveal rudimentary information processing. Van Honk et al. (2000) and Van Honk, Tuiten, De Haan, Van den Hout, and Stam (2001), for instance, have administered masked emotional Stroop tasks, in which neutral and emotional facial expressions were presented for a very brief time interval followed by a colored mask. In these experiments, subjects simply had to name the color of the mask as fast as possible. Albeit forced-choice awareness checks afterwards objectively confirmed that the facial expressions were inaccessible to consciousness, depending on motivational stance, the subjects did respond differently in terms of latency time when the mask was preceded by emotional as compared to neutral facial expressions. These findings do not only provide evidence

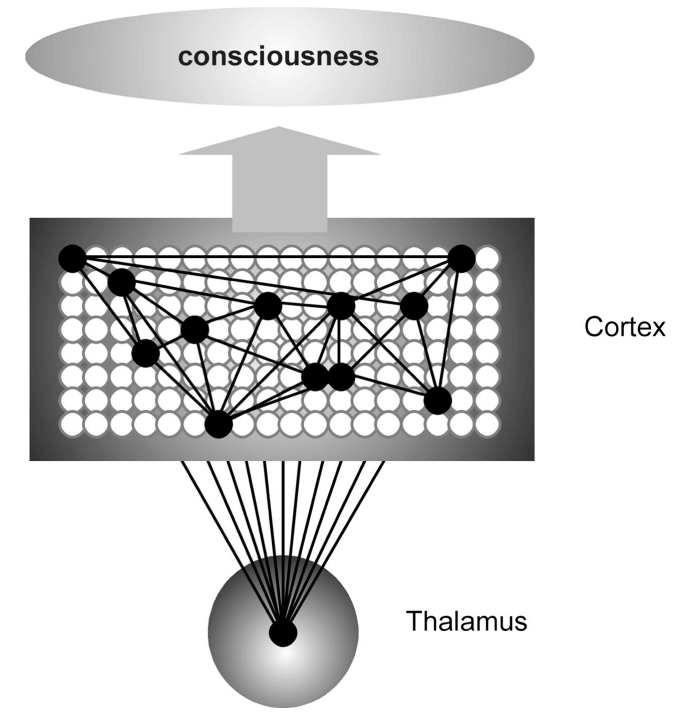

Fig. 1. Schematic representation of TCC networks realizing a transient functional connectivity pattern associated with consciousness. 
for Block's second explanation, but also signify a special role for emotion in consciousness. Indeed, research in the field of the affective neurosciences has provided substantial physiological and behavioral evidence for differential processing of emotional compared to neutral facial expressions (for a review, see Vuilleumier, 2002). Emotional facial expressions have evolved as refined communicative signals to promote fitness and survival. In the primates' social system, affective laden faces are thus very important from an evolutionary point of view (Darwin, 1872).

To get a firmer grip on neocortical dynamics, thalamo-cortical and cortico-cortical EEG coherence can provide for valuable insights regarding functional connectivity (Lubar, 1997). EEG coherence is a statistical measure that indexes the degree to which the electroencephalogram (EEG) signals from two cortical areas are correlated (Nunez et al., 1997). This measure of correlation is argued to reflect a state of functional connectivity (Thatcher, Krause, \& Hrybyk, 1986), which can therefore be used to determine connectivity patterns across the cortical surface. More specifically, low EEG coherences are associated with functional disconnection between thalamocortico-cortical regions, whereas high EEG coherences are linked to thalamo-cortico-cortical interaction. Cross-spectra for the different frequency bands can be calculated and normalized by the autospectra to compute EEG coherence, according to the following equation:

$$
\left|R_{x y}(e)\right|^{2}=\frac{\left|G_{x y}(e)\right|^{2}}{G_{x x}(e) * G_{y y}(e)} .
$$

In the equation, $G_{x x}(e), G_{y y}(e)$, and $G_{x y}(e)$ are values of the auto- and cross-spectra at a given frequency band $e$, respectively. Furthermore, since functional connectivity is generated by means of reciprocal interactions in the TCC networks, it makes a suitable method for investigating the electrophysiological correlates of GWT inspired architectures. Although we argue that EEG coherence estimation is informative regarding functional brain states in terms of functional connectivity, the relationship between EEG coherence estimates and functional connectivity has been deemed problematic (e.g., Breakspear, 2002; Fingelkurts et al., 2003; Horwitz, 2003; Kaplan \& Shishkin, 2000). Furthermore, since EEG signals show a finite correlation even when recorded from separate subjects, one must be cautious when interpreting EEG coherence as a measure of functional connectivity (Shaw, 1984). In a more broader sense, EEG coherence can nevertheless be interpreted as a measure of functional connectivity.

On the premise that unconscious information can influence conscious experience and that emotional valence is important in amplifying this effect, the present study investigated functional connectivity after displaying masked and unmasked angry, happy, and neutral facial expressions. Since unconscious affect information is argued to shape consciousness, it was hypothesized that the normally occurring reductions in EEG coherence after masked exposure would not occur when emotion was involved.

\section{Methods}

\subsection{Subjects}

Seventeen right-handed healthy volunteers (means \pm SD age, $21.6 \pm 1.9$ years) participated. None of the subjects had a history of psychiatric or neurological conditions and had normal or 
corrected-to-normal vision. Informed consent was obtained and participants received payment for taking part in the study. The protocol was approved by the Local Ethical Committee of Faculty of Social Sciences.

\subsection{Procedure}

The Ekman and Friesen (1976) pictures of facial affect were used as stimuli. In the experiment, 10 different faces were used, each displaying neutral, angry, and happy facial expressions. For constructing slides of masks we randomly cut, re-assembled, and re-photographed pictures of faces. Subjects randomly viewed 30 angry, neutral, and happy facial expressions (90 trials), which were presented for $14 \mathrm{~ms}$ followed by a mask $(1500 \mathrm{~ms})$, thereby effectively precluding conscious awareness of the emotional content of the face. The masked condition was followed by an awareness check to check whether the faces exposed during the masked remained unseen by the viewer. The check contained 30 emotional faces, i.e., angry, happy, and neutral, and were again presented for $14 \mathrm{~ms}$ followed by a mask. In a three-alternative forced choice procedure, the objective for the viewer was to indicate by a after each masked presentation whether the mask was preceded by an angry, happy or neutral facial expression. Ten faces of each category were randomly selected from the pool used during the actual subliminal passive viewing task. The experiment was concluded by randomly displaying 30 unmasked angry, neutral, and happy facial expressions (90 trials), that is a $14 \mathrm{~ms}$ mask followed by the facial expression (1500 ms).

\subsection{EEG recordings and analysis}

EEGs were recorded from eight electrodes, i.e., F3, F4, P3, P4, Fz, Cz, Pz, and Oz scalp positions, ${ }^{1}$ using an Electro-Cap with $\mathrm{Ag} / \mathrm{AgCl}$ electrodes (Neurosoft, Inc.), according to the International 10-20 System. EEG signals were referenced to an electrode placed behind the subject's right mastoid. The choice of the reference has been a longstanding issue in electrophysiological studies (e.g., Nunez et al., 1997; Picton et al., 2000; Reilly, 1999). Various proposals have been put forward to minimize the influence of reference, but no agreement on an optimal solution for the reference problem has been reached yet (Hagemann, Naumann, \& Thayer, 2001). The mastoid reference is often used to maximize inter-electrode distance and to avoid mixed activity from two different scalp areas. This montage is based on the assumption that unlike, for instance, vertex referencing the mastoid site picks up no or minimal electrical activity. Although, the reference electrode ought to be "silent," this is hard to accomplish. One of the solutions is to use a scalp site with minimal activity (Hagemann, Naumann, Becker, Maier, \& Bartussek, 1998), which was done in the present study.

\footnotetext{
${ }^{1}$ The choice of these electrode positions builds on Block's theoretical accounts on the differences between phenomenal and reflexive consciousness, and consciousness, as envisioned by, for instance, Crick and Koch (1998). Undoubtedly, consciousness extends beyond the visual modality and is present even when the sensory system is not innervated. Dehaene and Naccache (2001) have argued that consciousness allows the organism to act more on internal representations, hence more independently from the external environment, thereby increasing evolutionary fitness. Neurophysiologically, the neural global workspace might therefore encompass a more modality independent brain space, suggesting a special role for the cortical association areas, such as the prefrontal and parietal cortex.
} 
For the purpose of artefact scoring, vertical (VEOG) and horizontal (HEOG) eye movements were recorded. $\mathrm{Ag} / \mathrm{AgCl}$ electrode pairs (bipolar) were placed at the supra- and suborbit of the right eye and at the external canthi of each eye. ECI EEG gel was used for both EEG and EOG and all electrode impedances were less than 5,000 $\Omega$. An acquisition amplifier (Ampligraph) was used to filter incoming signals (low-pass cut-off frequency $70 \mathrm{~Hz}$; time constant $3 \mathrm{~s}$ ). For the EEG recordings NeuroScan software (El Paso, Texas) was used. Amplification was set at 20,000 for both the EEG and EOG leads, and the sample rate was $250 \mathrm{~Hz}$. All EEG coherences (0-1024 ms post-stimulus) between $\mathrm{Fz}, \mathrm{Cz}, \mathrm{Pz}, \mathrm{Oz}, \mathrm{P} 3, \mathrm{P} 4, \mathrm{~F} 3$, and F4 scalp sites were calculated and averaged to compute an index of overall EEG coherence in the $\delta(1-3 \mathrm{~Hz}), \theta(4-7 \mathrm{~Hz}), \alpha(8-12 \mathrm{~Hz})$, $\beta(13-30 \mathrm{~Hz})$, and $\gamma(31-50 \mathrm{~Hz})$ bandwidth.

\section{Results}

Non-parametric binomial tests for deviation (33\%) showed that the masking procedure had been effective and that none of participants were able to consciously identify the masked facial expressions $(P>.80)$. Two separate MANOVAs for the lower ( $\delta$ and $\theta$ range) and higher $(\alpha$ and $\beta$ range) frequency EEG coherences were performed with Task (masked, unmasked), Emotion (neutral, anger, and happy) as within-subject factors. A main Task effect for the lower frequency bands was found $[F(1,16)=4.45, P=.05]$, indicating overall lower EEG coherences in the unconscious (means $\pm \mathrm{SEM}, 0.51 \pm 0.01$ ) compared to the conscious perception condition (mean$\mathrm{s} \pm \mathrm{SEM}, 0.52 \pm 0.01)$. Furthermore, a significant Task $\times$ Emotion interaction effect was found $[F(2,15)=4.1, P<.038]$. Since no Task $\times$ Emotion $\times$ Band interaction $(P=.75)$ was obtained, $\delta$ and $\theta$ frequency EEG coherences were averaged and post hoc Bonferroni-corrected pairedsampled $t$ tests were applied to further test differences in EEG coherence of facial expressions between task conditions. Significant lower EEG coherences were found for the unmasked as compared to the masked neutral facial expressions in the $[t(17)=-3.48, P=.009]$, whereas the EEG coherences between the masked and unmasked emotional facial expressions were not statistically different (both $P$ 's $>0.61$ ). The MANOVA for the high frequency EEG coherences did not yield any statistical significant main task or interaction effects (all $P$ 's $>0.32$ ).

The present findings demonstrate that although lower frequency EEG coherence is overall reduced and in particular for the neutral facial expression in the masked condition, the lower frequency EEG coherences do not significantly change when masked and unmasked emotional facial expression are compared. Fig. 2 displays the collapsed $\delta$ and $\theta$ EEG coherences in the lower frequency bandwidth and the collapsed $\alpha, \beta$, and $\gamma$ EEG coherences in the higher frequency bandwidth for the masked and unmasked neutral, angry, and happy facial expressions. ${ }^{2}$

\footnotetext{
${ }^{2}$ Fig. 2 suggests that EEG coherence is largest for neutral conscious stimuli, but reduced for emotional conscious stimuli. Although, the differences in functional connectivity between the conscious facial expressions were not statistically significant $[F(2,15)=1.26, P=.31]$, this pattern is compatible with the view of Alexandrov (2002), who suggests an inverse relationship between consciousness and emotion. Furthermore, since the alleged origin of emotion stems from unconscious subcortical structures (Panksepp, 1998) and emotional facial expressions are vital in human social systems (Darwin, 1872), the neural global workspace might be especially tuned for such emotion laden information.
} 


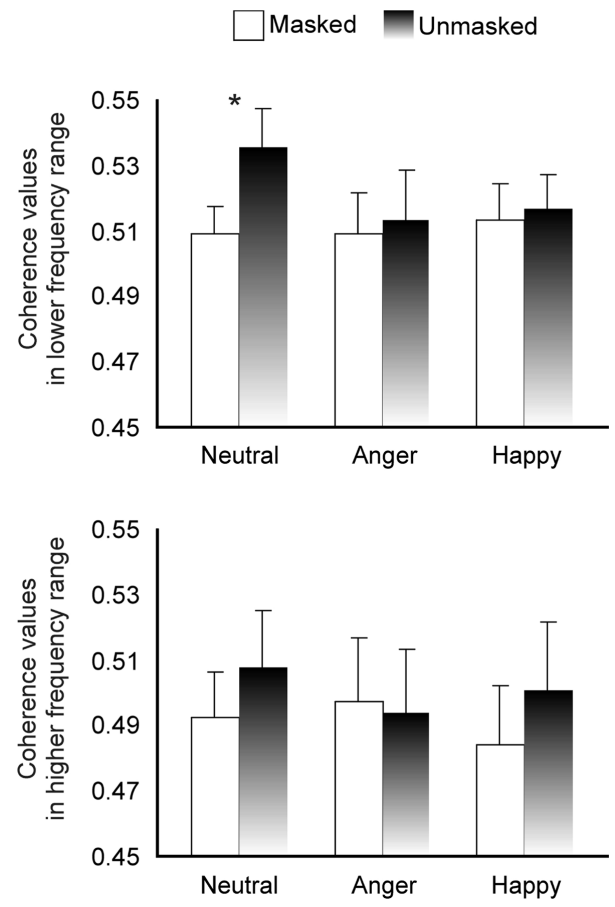

Fig. 2. Mean \pm SEM overall EEG coherence in the low frequency range for the unconscious (masked) and conscious (unmasked) neutral and emotional facial expressions. ${ }^{*} P=.003$.

\section{Discussion}

According to Crick and Koch (1998), consciousness involves distributed brain regions constructing a multilevel, explicit, symbolic interpretation of a stimulus, that is functionally connected in time (synchronicity). Furthermore, emotion is suggested to play a role in the modulation of these processes even on a unconscious level. In the present study, functional connectivity was therefore investigated after displaying neutral and emotional facial expressions in masked and unmasked conditions. It should however be noted that formally functional connectivity is defined as the temporal correlation between spatially remote neurophysiological events (Friston, 1994), such as for instance masked and unmasked emotional facial expression. Since EEG coherence indicates only the linear statistical link between EEG signals across different scalp sites, it is possible to identify the involvement of the different brain regions, but information regarding their specific contribution is not available (Fingelkurts \& Fingelkurts, 2001). Functional connectivity might best be viewed as a global functional state of the brain. The current finding of lower EEG coherence in the masked condition fits with a previous study which demonstrated a correlation between conscious perception and increased cortical EEG coherence (Srinivasan, Russell, Edelman, \& Tononi, 1999). Thus, further experimental support is found for the neural global workspace architecture, indicating that consciousness is linked to a level of EEG coherence.

Although, synchronization or EEG coherence in the $\gamma$ frequency range is argued to reflect electrophysiological properties of consciousness (Keil, Müller, Ray, Gruber, \& Elbert, 1999; 
Meador, Ray, Echauz, Loring, \& Vachtsevanos, 2002) presently modulations in the lower frequency bandwidth were demonstrated. Albeit Crick and Koch (2003) have recently argued that $\gamma$ frequency band might to be critical to consciousness, it is important to distinguish between findings derived from studying local networks (e.g., visual cortex) that are specifically tuned to process information at higher frequency ranges (Crick \& Koch, 1997) and findings from global distributed networks between which convey information at lower frequency bandwidths (Lubar, 1997; Silberstein, 1995). The present data suggest that a level of EEG coherence normally indicating consciousness can be reached when stimuli are presented below the psychophysical threshold of conscious awareness. These findings concur with a neuropsychological study by Driver and Vuilleumier (2001). Driver and Vuilleumier (2001) displayed schematic neutral, angry, and happy facial expressions to parietal lesion patients with hemineglect. On bilateral presentation trials, contralesional happy and angry facial compared to neutral facial expressions showed significantly less extinction, suggesting a processing advantage for affect laden face stimuli.

Moreover, because changes in EEG coherence in the lower EEG bandwidths have been associated with subcortical drive (Locatelli, Cursi, Liberati, Franceschi, \& Comi, 1998; Schutter \& Van Honk, 2004), the current findings in the lower frequency range are notable. The subcortical structures of the brain are heavily involved in motivation and emotion (Panksepp, 1998), thus the lower frequency bandwidth might serve as a carrier wave for affective information transfer from subcortical structures to the cortical workspace. Sustained EEG coherence after the display of masked emotional facial expressions might be viewed as the inaccessible instantiation of reflexive consciousness. Block (2001) already argued that there is no a priori reason to believe that unconsciously presented stimuli cannot have qualitative properties differentially influencing the receiving system. Consciousness might better be described in terms of a continuum, which does not solely rely on passing a certain threshold per se. When emotion come into play this continuum might on the neuroanatomical level be shaped by unconscious affective subcortical processes interacting with the neuronal workspace capable of generating different levels of awareness (Ledoux, 1996). This notion is further evidenced by the earlier mentioned study by Driver and $\mathrm{Vu}-$ illeumier (2001), which provided evidence for reduced contralesional extinction of emotional facial expressions in patients with parietal neglect. Furthermore, Edelman (2001) has suggested that in particular ongoing reentrant signaling (reverberation) across multiple connected brain areas or loci is the foundation for conscious awareness. In the present study, the reverberation across the different regions might be interpreted in terms of sustained EEG coherence of masked as compared to unmasked emotional facial expressions. It is therefore suggested that the current absolute level of EEG coherence in the global working space can be considered as an electrophysiological correlate for affective content of phenomenality. To re-direct the cortical focus to more subcortical contributions in discussing the neurocircuitry underlying different forms of consciousness, the subcortical innervated arousal responses in terms of efferent mescenphale cholinergic projections to the cortical workspace via the thalamus (Parvizi \& Damasio, 2001; Arai, Tanaka, Pascual-Marqui, \& Hirata, 2003) might boost the cortex in order to be able to respond more readily if necessary (action preparedness). Koriat and Levy-Sadot (2003), for example, discussed unconscious priming effects on strategy selection and consequently introduced the theoretical notion of implicit meta-cognitive monitoring, which in the present case would be colored by affective content. In accordance with previous findings it is concluded that EEG coherence might indeed serve as an electrophysiological index for different levels of consciousness. 
The present findings do not only show that the receptive field of the cortex extends beyond the regular sensory innervations, but it also provides the first evidence for EEG coherence being gated by affective content when stimuli are presented outside conscious awareness.

Since unconscious percepts can be (1) phenomenally and reflexively innate or (2) phenomenally conscious but reflexively unconscious (Block, 2001), the current findings provide additional support for Block's second explanation in which the brain is able to make a qualitative distinction despite the information is not accessible to the perceiver.

In sum, although the role of emotion in consciousness has been acknowledged (e.g., Damasio, 1994; Panksepp, 1998), cognitive based architectures have dominated the philosophy of mind. With the advent of the modern neuroimaging techniques the body-mind problem has shifted towards the unravelment of the NCC, and evidence in favor of GWT like architectures has been accumulating (Baars, 2002). In addition, we would like to argue that the emergence of consciousness was an adaptive necessity for survival extending far beyond signal detection at the level of the visual cortex. Further research into the role of emotion in the GWT might provide for valuable insights into the functional significance and neural representation of consciousness.

\section{Acknowledgments}

We thank the two anonymous reviewers for their valuable comments on earlier versions of this draft. This study was sponsored by an Innovational Research Grant (No. 016-005-060) from the Netherlands Organization for Scientific Research (NWO).

\section{References}

Alexandrov, Y. I. (2002). Neuronal specializations, emotion and consciousness within culture. In Toward a Science of Consciousness (p. S405). Tucson: University of Arizona.

Arai, M., Tanaka, H., Pascual-Marqui, R. D., \& Hirata, K. (2003). Reduced brain electric activities of frontal lobe in cortical cerebellar atrophy. Clinical Neurophysiology, 114, 740-747.

Baars, B. J. (1988). A cognitive theory of consciousness. Cambridge: Cambridge University Press.

Baars, B. J. (2002). The conscious access hypothesis: Origins and recent evidence. Trends in Cognitive Sciences, 6, 47-52.

Baars, B. J., \& Franklin, S. (2003). How conscious experience and working memory interact. Trends in Cognitive Sciences, 7, 166-172.

Block, N. (1995). Paradox and cross purposes in recent work on consciousness. Cognition, 79, $197-219$.

Block, N. (1996). How can we find the neural correlate of consciousness. Trends in Neurosciences, 19, 456-459.

Block, N. (2001). Paradox and cross purpose in recent work on consciousness. Cognition, 79, 197-219.

Breakspear, M. (2002). Nonlinear phase desynchronization in human electroencephalographic data. Human Brain Mapping, 15, 175-198.

Buck, R. (1999). The biological affects: A typology. Psychological Review, 106, 301-336.

Crick, F., \& Koch, C. (1997). Towards a neurobiological theory of consciousness. In N. Block, O. Flanagan, \& G. Güzeldere (Eds.), The nature of consciousness: Philosophical debates (pp. 277-292). Massachusetts: MIT Press.

Crick, F., \& Koch, C. (1998). Consciousness and neuroscience. Cerebral Cortex, 8, 97-107.

Crick, F., \& Koch, C. (2003). A framework for consciousness. Nature Neuroscience, 6, 119-126.

Damasio, A. (1994). Descartes' error: Emotion, reason, and the human brain. New York: Putnam Press.

Darwin, C. (1872). The expression of the emotions in man and animals. New York: D. Appleton and Company.

Dehaene, S., Kerszberg, M., \& Changeux, J. P. (1998). A neuronal model of a global workspace in effortful cognitive tasks. Proceedings of the National Academy of Sciences of the United States of America, 95, 14529-14534. 
Dehaene, S., \& Naccache, L. (2001). Towards a cognitive neuroscience of consciousness: Basic evidence and a workspace framework. Cognition, 79, 1-37.

Dehaene, S., Sergent, C., \& Changeux, J. P. (2003). A neuronal network model linking subjective reports and objective physiological data during conscious perception. Proceedings of the National Academy of Sciences of the United States of America, 100, 8520-8525.

Driver, J., \& Vuilleumier, P. (2001). Perceptual awareness and its loss in unilateral neglect and extinction. Cognition, 79 , $39-88$.

Edelman, G. (2001). Consciousness: The remembered present. Annals of the New York Academy of Sciences, 929, 111-122.

Ekman, P., \& Friesen, W. (1976). Pictures of facial affect. Palo Alto: Consulting Psychologist Press.

Fingelkurts, A. A., \& Fingelkurts, A. A. (2001). Operational architectonics of the human brain biopotential field: Towards solving the mind-brain problem. Brain and Mind, 2, 261-296.

Fingelkurts, A. A., Fingelkurts, A. A., Krause, C., Kaplan, A., Borisov, S., \& Sams, M. (2003). Structural (operational) synchrony of EEG alpha activity during an auditory memory task. Neuroimage, 20, 529-542.

Friston, K. J. (1994). Functional and effective connectivity in neuroimaging: A synthesis. Human Brain Mapping, 2, 5678.

Hagemann, D., Naumann, E., Becker, G., Maier, S., \& Bartussek, D. (1998). Frontal brain asymmetry and affective style: A conceptual replication. Psychophysiology, 35, 372-388.

Hagemann, D., Naumann, E., \& Thayer, J. F. (2001). The quest for the EEG reference revisited: A glance from brain asymmetry research. Psychophysiology, 38, 847-857.

Horwitz, B. (2003). The elusive concept of brain connectivity. Neuroimage, 19, 466-470.

Kaplan, A., \& Shishkin, S. L. (2000). Application of the change-point analysis to the investigation of the brain's electrical activity. In B. E. Brodsky \& B. S. Darhovsky (Eds.), Nonparametric statistical diagnosis: Problems and methods (pp. 333-388). Dordrecht: Kluwer Academic Publishers.

Keil, A., Müller, M. M., Ray, W. J., Gruber, T., \& Elbert, T. (1999). Human gamma band activity and perception of gestalt. Journal of Neuroscience, 19, 7152-7161.

Kelso, J. A. S. (1984). Phase transitions and critical behavior in human bimanual coordination. American Journal of Physiology, 15, R1000-R1004.

Koriat, A., \& Levy-Sadot, R. (2003). Conscious and unconscious metacognition: A rejoinder. Consciousness and Cognition, 9, 193-202.

Ledoux, J. E. (1996). The emotional brain. New York: Simon and Schuster.

Llinas, R., Ribrary, U., Contreras, D., \& Pedroarena, C. (1998). The neuronal basis for consciousness. Philosophical Transactions of the Royal Society of London B, 353, 1841-1849.

Locatelli, T., Cursi, M., Liberati, D., Franceschi, M., \& Comi, G. (1998). EEG coherence in Alzheimer's disease. Electroencephalography and Clinical Neurophysiology, 106, 229-237.

Lubar, J. F. (1997). Neocortical dynamics: Implications for understanding the role of neurofeedback and related techniques for the enhancement of attention. Applied Psychophysiology and Biofeedback, 22, 111-126.

Meador, K. J., Ray, P. G., Echauz, J. R., Loring, D. W., \& Vachtsevanos, G. J. (2002). Gamma coherence and conscious perception. Neurology, 59, 847-854.

Nunez, P. L, Srinivasan, R., Westdorp, A. F., Wijesinghe, R. S., Tucker, D. M., Silberstein, R. B., \& Cadusch, P. J. (1997). EEG coherency I: Statistics, reference electrode, volume conduction, Laplacians, cortical imaging, and interpretation at multiple scales. Electroencephalography and Clinical Neurophysiology, 103, 499-515.

Oately, K. (1992). Best laid schemes: The psychology of emotions. Cambridge: Cambridge University Press.

Panksepp, J. (1998). Affective neuroscience: The foundations of human and animal emotions. New York: Oxford University Press.

Parvizi, J., \& Damasio, A. (2001). Consciousness and the brain stem. Cognition, 79, 135-159.

Picton, T. W., Bentin, S., Berg, P., Donchin, E., Hillyard, S. A., Johnson, R., Jr., Miller, G. A., Ritter, W., Ruchkin, D. S., Rugg, M. D., \& Taylor, M. J. (2000). Guidelines for using human event-related potentials to study cognition: Recording standards and publication criteria. Psychophysiology, 37, 127-152.

Reilly, E. L. (1999). EEG recording and operation of the apparatus. In E. Niedermeyer \& F. Lopes da Silva (Eds.), Electroencephalography: Basic principles, clinical applications, and related fields (fourth ed., pp. 122-142). Baltimore: Williams and Wilkins. 
Schutter, D. J. L. G., \& Van Honk, J. (2004). Decoupling of midfrontal delta-beta oscillations after testosterone administration. International Journal of Psychophysiology, 53, 71-73.

Shaw, J. C. (1984). Correlation and coherence analysis of the EEG: A selective tutorial review. International Journal of Psychophysiology, 1, 255-266.

Silberstein, R. B. (1995). Neuromodulation of neocortical dynamics. In P. L. Nunez (Ed.), Neocortical dynamics and human EEG rhythms (pp. 591-627). New York: Oxford University Press.

Srinivasan, R., Russell, D. P., Edelman, G. M., \& Tononi, G. (1999). Increased synchronization of neuromagnetic responses during conscious perception. Journal of Neuroscience, 19, 5435-5448.

Thatcher, R. W., Krause, P. J., \& Hrybyk, M. (1986). Cortico-cortical associations and EEG coherence: A twocompartmental model. Electroencephalography and Clinical Neurophysiology, 64, 123-143.

Tomkins, S. S. (1982). Affect theory. In P. Ekman (Ed.), Emotions in the human face (second ed., pp. 353-395). Cambridge: Cambridge University Press.

Tononi, G., \& Edelman, G. M. (1998). Consciousness and complexity. Science, 282, 1846-1851.

Van Honk, J., Tuiten, A., Van den Hout, M., Koppeschaar, H., Thijssen, J., De Haan, E., \& Verbaten, R. (2000). Conscious and preconscious selective attention to social threat: Different neuroendocrine response patterns. Psychoneuroendocrinology, 25, 577-591.

Van Honk, J., Tuiten, A., De Haan, E., Van den Hout, M., \& Stam, H. (2001). Attentional biases for angry faces: Relationships to trait anger and anxiety. Cognition and Emotion, 15, 279-297.

Vuilleumier, P. (2002). Facial expression and selective attention. Current Opinion in Psychiatry, 15, 291-300.

Zeman, A. (2001). Consciousness. Brain, 124, 1263-1289. 